\title{
Répartition et flux de matière organique dans un écosystème à Pinus pinea $\mathrm{L}$.
}

\author{
Maurice RAPP \\ C.N.R.S.-C.E.P.E. - L. Emberger \\ B.P. 505I - F 34033 Montpellier
}

\begin{abstract}
Résumé
La distribution et les flux de matière organique ont été étudiés dans un peuplement de pins pignons de la côte méditerranéenne française, âgé d’une trentaine d'années. Le peuplement se compose de 800 tiges à l'hectare, de $10,5 \mathrm{~m}$ de hauteur en moyenne et de surface terrière moyenne de $320 \mathrm{~cm}^{2}$.

Le stock de matière organique du sitc se monte à $377 \mathrm{t}$ ha 1 dont $179 \mathrm{t} \mathrm{ha}^{-1}$ représentent la phytomasse aérienne et racinaire. 158 t ha 1 sont constitués de matériel organique "mort", décomposé en nécromasse, litière au sol et matière organique du sol.

La production nete de la station est de $18,6 \mathrm{t} \mathrm{ha-1}^{-1}$, dont 7,2 a ha 1 de matériel pérenne. La retombée annuclle de litière est de 7,9 tha 1 . La matière organique da sol šélève à 105 t ha 1 sur un mètre de profondeur, alors que 41,6 thal 1 de litière sont immobiliscés à la strfface du sol.
\end{abstract}

\section{Introduction}

Les végétaux supérieurs autotrophes possèdent la faculté unique de constituer, a partir de l’énergie solaire, du gaz carbonique de l'air. de l'eau et des éléments minéraux du sol, un matériel énergétique nouveau, leur propre matière organique. Celle-ci, consomméc ou redistribuée, est à la base de la vie animale el microbienne de l'ćcosystème el constituc un facteur primordial de l'évolution du sous-système sol.

l.a répartition de cette matière organique et ses transferts dans l'écosystème constituent ce que l'on appelle le cycle du carbone ou le cycle de la matière organique selon que l'approche énergétique ou l'approche quantitative et pondérale est privilégiéc. Alors que dans les formations herbacées on étudie souvent le cycle du carbone, on s'intéresse dans les écosystèmes forestiers, à cause des difficultés méthodologiques inhérentes au matériel d’étude, au cycle global de la matière organique.

Celui-ci peut être décomposé en deux grandes phases : une phase anabolique, comportant la synthèse de substances nouvelles, puis leur transfert el leur répartition 
à travers l'organisme vers les organes en croissance. A ce stade de production succède une séric d'étapes cataboliques, débutant par la sénescence des organes non pérennes, essenticllement photosynthétiques. leur retour au sol, suivi de leur décomposition et de leur inclusion partielle dans la fraction organique du sol. Une ultime minéralisation verra le retour à l'état minéral sous forme de gaz carbonique, d'éléments minéraux et d'eau.

De nombreuses éludes ont été consacrées au cours des deux dernières décennies à l'évaluation du cycle de la matière organique dans les systèmes forestiers, soit comme objet d'étude principale, soit comme partic intégrante de l'étude plus vaste des cycles biogéochimiques. Aux données collectées et inventoriées par Rodin \& BAzIlevici (1967), l'on peut ajouter les résultats plus récents, obtenus dans le cadre du programme IPB, qui ont été synthétisés par CoLI \& RAPP (1980) en ce qui concerne les recherches les plus marquantes et les plus complètes.

Le présent travail représente la synthèse de la répartition el de l'évolution de la matière organique dans un écosystème à pin pignon (Pinus pinea L.), implanté sur des dunes du littoral méditerranéen. Il constitue un maillon important d'un projet plus large visant également à définir et à quantifier le fonctionnement de cet écosystème au niveau du cycle et de l'économic de l'eau (Irrahim el al., 1982) et des éléments minćraux biogènes (RAp' et al.. 1979). De par la localisation du peuplement étudié en région méditerranéenne, les résultats obtenus permettent également d'étoffer un peu plus les connaissances propres aux écosystèmes ligneux méditerranéens, qui sont encore relativement réduites par rapport à celles relatives aux systèmes forestiers des zones boréales et tempérées, voire tropicales el ćquatoriales.

\section{Le site étudié}

La station de Pinus pinea L. qui fait lobjet de ce travail est localiséc sur les bords de la Méditerranée, à $15 \mathrm{~km}$ de la mer, aire dans laquelle cette essence est la plus abondante à l'ouest du Rhône.

Le peuplement couvre 30 ha et les caractéristiques dendrométriques, établies pour un placeau d'étude de $2400 \mathrm{~m}^{2}$ et exprimées à l'hectare, sont les suivantes :

$\begin{array}{lc}\text { Nombre d'arbres par hectare } & 800 \\ \text { Surface terrière par hectare } & 39,9 \mathrm{~m}^{2} \\ \text { Hauteur de l'arbre moyen } & 10,4 \mathrm{~m} \\ \text { Circonférence moyenne par arbre } & 63,4 \mathrm{~cm}\end{array}$

Pinus pinea occupe la totalité de la strate arborescente, alors que dans la strate arbustive l'on trouve également Quercus lanuginosa Lamk., Robinia pseudoacacia L., Populus alba L., Ulmus campestris L., Phillyrea angustifolia L., Rhannus alaternus L.

Ce peuplement résultant d'une régénération naturelle après la coupe de la quasi totalité des arbres en 1944, est inéquienne comme le laisse pressentir l'histogramme des classes de circonférence (fig. 1).

Une étude détaillée de 61 individus fait apparaître cinq classes d'âge : 15 ans, 20-21 ans, 26-27 ans, 30 ans et $3 \tilde{3}$ ans. 


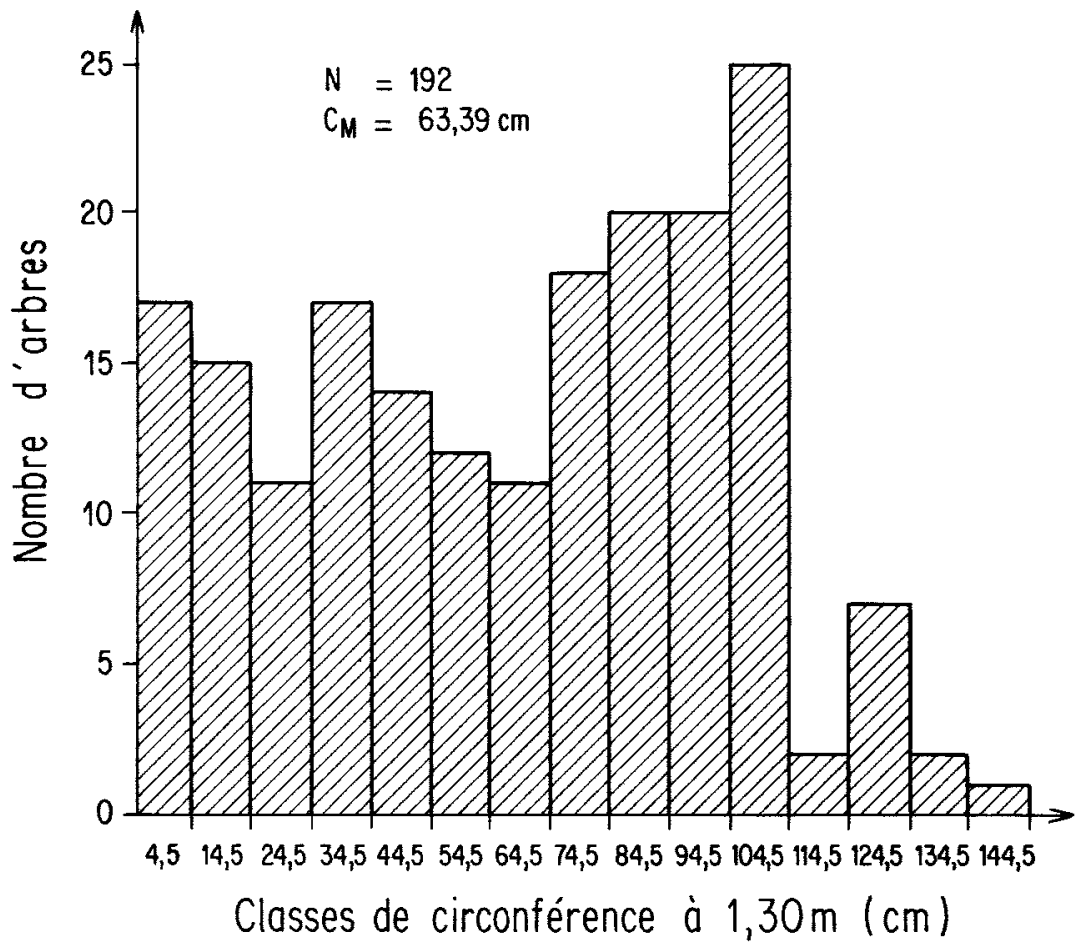

FIG. 1

Histogramme de fréquence des circonférences à $1,30 \mathrm{~m}$ du tronc des différents individus du placeau d'éiude

$(N=$ nombre d'individus $; C M=$ circonférence moyenne $)$.

Girth distributions $(D B H)$ of the trees from the study site $(N=$ number of trees, $C M=$ mean girth).

Le climat est de type méditerranéen subhumide à hiver tempéré (EMbERGER, 1955). Les caractéristiques climatiques de la station météorologique la plus proche $(8 \mathrm{~km})$ sont les suivantes :

\begin{tabular}{|c|c|c|}
\hline Précipitations annuelles & & $648 \mathrm{~mm}$ \\
\hline Température moyenne annuelle & & $14,2^{\circ} \mathrm{C}$ \\
\hline Température minimale moyenne & $\begin{array}{l}\text { juillet } \\
\text { décembre }\end{array}$ & $\begin{array}{r}17,6^{\circ} \mathrm{C} \\
3,2^{\circ} \mathrm{C}\end{array}$ \\
\hline Température maximale moyenne & $\begin{array}{l}\text { juillet } \\
\text { décembre }\end{array}$ & $\begin{array}{r}27,7^{\circ} \mathrm{C} \\
9,8^{\circ} \mathrm{C}\end{array}$ \\
\hline
\end{tabular}

Le sol s'est formé sur d'anciennes dunes stabilisées par les arbres. Il se compose d'une litière d'épaisseur variable $(7$ à $12 \mathrm{~cm})$, reposant sur un horizon $A_{1}$ de 0 à $20 \mathrm{~cm}$ d'épaisseur, recouvrant lui-même un horizon $C$ sableux calcaire. Entre -20 
et $-80 \mathrm{~cm}$ l'on trouve, dans certaines zones un niveau relativement plus riche en substances organo-minérales d'une vingtaine de $\mathrm{cm}$ d'épaisseur, reliquat du fond d'un ancien marais recouvert par le sable dunaire. D'après la classification irançaise des sols, c'est un régosol peu évolué, sur sable d'origine colienne (IввАнгм \& Rapl, 1979).

Une nappe phréatique à faible profondeur, assure à la végétation une alimentation permanente en cau (IBRAHIM, 1979).

\section{- Méthodes d'étude}

Les méthodes concernant les évaluations de la phytomasse, de la productivité et de l'accroissement de la biomasse pérenne ayant été décrites par CABANLTTES (1979), Cabanettes \& Rapl (1978, 1981 b), seules les méthodes d'études des transferts de la matière organique à travers l'écosystème seront analysées en détail.

\section{Evaluation des compartiments}

\subsection{La phytomasse totale des arbres}

Elle a été déterminée par une approche mixte (Duvignenud, 1967 ; Niwbould, 1967 ; RiEDACKLR, 1968) comportant à la fois des mesures des biomasses et des caractéristiques dendrométriques d'un certain nombre d'arbres abattus et le calcul d'équations de régression : circonférence du trone à $1,30 \mathrm{~m}$ et biomasse totale ou partielle de ces arbres. Cés équations sont alors appliquées aux caractéristiques dendrométriques des arbres du peuplement restés sur pied.

Ces équations, doublement logarithmiques sont les suivantes (CABANETTEs, 1979) :

- $\log$ phytomasse aćrienne $=2,62521 \log$ circonférence $1,30 \mathrm{~m}+0,33501$ $(r=0,931)$

- $\log$ biomasse feuille $\quad=2,62178 \log$ circonférence $1,30 \mathrm{~m}-0,75075$ $(\mathrm{r}=0,948)$

$-\log$ biomasse ligneuse $=2,64268 \log$ circonférence $1,30 \mathrm{~m}+0,26253$ $(\mathrm{r}=0,997)$

$-\log$ biomasse racines $\quad=1,71552 \log$ circonférnece $1,30 \mathrm{~m}+1,23008$ $(r=0.980)$

\subsection{Le stock de litière au sol}

La litière accumuléc à la surface du sol constituc une couche uniforme. Sa masse a été déterminéc en 1975, 1978 et 1979 en prélevant chaque fois 10 échantillons, répartis sur l'ensemble du placeau d'étude, à l'aide d'un cmportc-pièce de $20 \mathrm{~cm}$ de diamètre (314 cm² de surface).

Chaque échantillon a été subdivisé d'après des critères morphologiques en $\mathrm{L}$, litière intacte provenant de l'annéc précédente, $F_{1}, F_{2}$ et $F_{3}$, niveaux correspondant à des stades de décomposition de plus en plus avancés et $A_{1} o_{1}$ correspondent à l'incorporation de la matière organique avec l'horizon minéral $\left(A_{1}\right)$ sous-jacent. 
Un dernier prélèvement a été effectué fin 1980. Bien que différent des trois précédents, il permet d'estimer, d'une façon moins détaillée, la litière accumuléc à la surface du sol de la station à cette époque.

Cette litière qui subsiste durant de longues périodes à la surface du sol. y subit une contamination par le sable sous-jacent, consécutive à l'activité de la faune fouisseuse. Elle s'accroît avec l'âge de la litière, et introduit une erreur importante dans l'estimation du stock. Pour s'affranchir de cette incertitude l'on a déterminé la perte au feu, par calcination, de chaque échantillon et de chaque fraction, ce qui permet de défalquer les contaminants d'origine minérale, échantillon par échantillon. En effet, la variabilité est trop importante à la fois entre les échantillons d"un même niveau et d'une année à l'autre comme l'indiquent les valeurs du tableau 1 concernant les tencurs $(p .100)$ en cendre, pour que l'on puisse se contenter d'unc correction globale.

\section{TABLEAU 1}

Teneur en cendres (p. 100 matière sèche) des différents prélèvements de litière au sol. Ash content (p. 100 dry weight) of the litter layer from the site.

\begin{tabular}{|c|c|c|c|c|c|}
\hline & $\mathbf{L}$ & $\mathrm{F}_{1}$ & $\mathbf{F}=$ & $F_{: ;}$ & $\mathrm{Ao}_{1}$ \\
\hline 1975 & $5,7 \pm 2,2$ & $10,3 \pm 4.2$ & $26.8+14,5$ & $50,0 \pm 12.6$ & $81,0 \pm 3,8$ \\
\hline 1978 & $23,9 \pm 14,1$ & $28,2 \pm 21,9$ & $38,0 \pm 21.1$ & $56,4 \pm 14,3$ & $78,6 \pm 17,4$ \\
\hline 1979 & $13.1 \pm 4.7$ & $29,3 \pm 15,2$ & $28,0 \pm 12,4$ & $52,0 \pm 14,7$ & $85,9 \pm 4,1$ \\
\hline 1980 & $5.9^{++} 2.1$ & & $14,4 \pm 2,5$ & $56,2 \pm 15,0$ & \\
\hline
\end{tabular}

\subsection{La matière organique dans le sol}

Le stock de matière organique du sol a été évalué sur une profondeur d'un mètre à partir de la densité apparente des différentes strates el de leur teneur en carbone. Cette étude (IBRAHIM \& RAPP, 1979) fournit les valeurs suivantes qui sont respectivement la moyenne de 14 profils pour la densité et de 3 profils pour la tencur en carbone :

\begin{tabular}{c|c|c}
\hline$\overline{\text { Profondeur }}(\mathrm{cm})$ & Densité apparente & Carbone $(\%)$ \\
\hline $0-20$ & 1,34 & 0,40 \\
$20-50$ & 1,39 & 0,20 \\
$50-70$ & 1,47 & 1,18 \\
$70-100$ & 1,44 & 0,17 \\
\hline
\end{tabular}

La teneur plus élevée de carbone entre 50 et $70 \mathrm{~cm}$ résulte de l'horizon plus riche en substances organo-minérales provenant du fond d'un ancien marais recouvert par des dunes plus récentes. 
A partir de ces valeurs, l'on peut calculer le poids at $\mathrm{m}^{2}$ ou à l'hectare de chacune des 4 strates, puis le poids de carbone qui y est inclus et le poids de matière organique $(\mathrm{C} \times 1,72)$.

Les valeurs des densilés déterminées de $10 \mathrm{~cm}$ en $10 \mathrm{~cm}$ présentent pour les 4 strates, un écart type inférieur à 8 p. 100.

\section{Evaluation des flux}

Les transferts de la matière organique de l'un à l'autre de ces compartiments se font essentiellement selon trois flux. Le premier qui est plutôt interne au comparm timent phytomasse, porte sur la production d'organes assimilateurs, puis la redistribution des photosynthétats dans l'organisme, done l'accroissement de la phytomasse pérenne et la fabrication d'organes reproducteurs. Le deuxième transfert se situe entre la phytocénose et le sol et concerne la retombée de litière. Le troisième lui succède directement; il représente la décomposition de la litière à la surface du sol.

\subsection{L'accroissement de la phytomasse}

Il a été estimé à partir de l'accroissement moyen annuel des trones en circonférences (CABANetTes, 1979), complété par l'analyse pondérale, après séparation des tissus et organes nouvellement formés. Ceci a permis de préciser la production des différentes fractions du bois (bois fort, bois fin), des pousses, des aiguilles et des organes reproducteurs. Ces observations effectuées sur un certain nombre d'arbres. ont été généralisées au peuplement en utilisant les relations entre les biomasses individuelles et la circonférence des troncs à $1,30 \mathrm{~m}$ (CABAnETtes \& RApr, 1981).

\subsection{La retombée de litière}

La récolte de litière a été effectuée mensuellement durant cinq années consécutives, de 1975 à 1979 sur le placeau d'étude de $2400 \mathrm{~m}^{2}$.

Sur cette surface, lon a disposé 28 paniers collecteurs de $0.25 \mathrm{~m}^{2}$ de surface chacun, placés selon un dispositif géométrique à partir d'un point choisi au hasard.

Après récolte, la litière a été séparée en cinq fractions : les aiguilles, une fraction ligneuse (bois) constituéc de fragments de branches et d'écorce, les inflorescences mâles et les fruits (cônes). Une dernière fraction, dénommée divers, regroupe le matériel végétal ne provenant pas du pin pignon.

Ces fractions ont été pesées après séchage à $80^{\circ}$ jusqu'à poids constant, et les résultats moyens cxprimés en $\mathrm{g} \mathrm{m}^{-2}$.

Une étude statistique de l'ensemble des résultats obtenus au cours d'une année par chaque collecteur donne la moyenne suivante :

$$
\mathrm{m}=56,5 \mathrm{~g} \mathrm{~m}^{-2}+21 \text { p. } 100
$$

Si l'on prend uniquement en compte les 21 paniers, situćs dans la fourchette de l'écart type, l'on obtient une nouvelle valeur moyenne de $57,3 \mathrm{~g} \mathrm{~m}^{-2}$ avec un écart type de 6,5 p. 100 seulement. 
Nous avons néanmoins conservé l'ensemble des 28 paniers collecteurs qui traduisent mieux l'hétérogénéité de la station.

\subsection{Décomposition de la litière}

Aucun dispositif particulier n'a été utilisé pour étudier ce flux in situ. Dans la discussion, nous nous baserons sur les données concernant le stock de litière au sol et de la retombée de litière pour évaluer son taux de décomposition.

\section{Résultats :}

3.1. La matière organique accumulée dans les différents compartiments de l'écosystème pin pignon

\subsection{La phytomasse}

A partir de l'étude démographique du peuplement, et des relations entre les caractéristiques des différents individus et de leurs poids, CabanetTes (1979) a estimé la biomasse totale du système à $178,8 \mathrm{tha}^{-1}$, se décomposant en $156,8 \mathrm{t} \mathrm{ha}^{-1}$ de phytomasse aérienne et $22 \mathrm{t} \mathrm{ha}^{-1}$ de phytomasse racinaire.

La partic aérienne peut elle-même se subdiviser en $12,7 \mathrm{tha}^{-1}$ de biomasse d'aiguilles et $144,1 \mathrm{t} \mathrm{ha}^{-1}$ de biomasse ligneuse dont 97,5 constituent le tronc et 46,6 les branches et les rameaux de la canopée.

A cette biomasse s'ajoute une nécromasse de l'ordre de $11,3 \mathrm{t}$ ha-1 formée essentiellement de branches mortes qui persistent durant un certain nombre d'années sur le tronc.

\subsection{La litière alı sol}

Le tableau 2 indique le stock global et fractionné de litière accumuléc à la surface du sol lors des quatre échantillonnages. Bien que celui de 1980 ait été entrepris dans un but différent, et ne peut donner lieu à des comparaisons qu'au niveau de la fraction de litière la plus récente ou de la litière globale, son inclusion permet d'affiner la moyenne.

Toutes ces valeurs représentent la matière organique sensu stricto, c'est-à-dire le matériel végétal échantillonné après déduction des cendres. Ceci permet d'éliminer la contamination minérale à partir du substrat qui est très variable, mais s'accroît avec le degré de décomposition de la litière, pour passer de 12,2 p. 100 en moyenne dans le niveau $\mathrm{L}$ à 89,9 p. 100 dans $\mathrm{Ao}_{1}$, avec de fortes variations d'une année sur l'autre.

Le rôle du substrat est encore accentué au niveau de la couche $A_{o_{1}}$ dans laquelle il est difficile de séparer avec précision le matériel appartenant strictement à la litière et la croûte la plus organique du sol. Mais en tenant compte de la den-

(*) Tous les résultats sont exprimés en poids sec, après séchage à $80^{\circ} \mathrm{C}$ à l'étuve jusqu'à poids constant. 
sité du sol (1,38), de sa teneur en carbone $(0,4 \mathrm{p} .100)$, l'on constate que l'inclusion d'une tranche de sol de l'ordre de 0.5 à $1 \mathrm{~cm}$ d'épaisseur ne représente qu'entre 0,5 à 1 t ha 1 de matière organique, ce qui est négligeable.

TABLI:AU 2

Stock total et fractionne de la litiere accumulèe à la surface du sol.

Résultats en $\mathrm{g} m$ ?. après déduction des cendres.

Partial and total amounts of litter accumulated on the soil surface.

Results expressed as gm? alter correction for the ash content.

\begin{tabular}{|c|c|c|c|c|c|c|c|}
\hline & & poid & des différer & s fraction & $m-2$ & & Total \\
\hline & L. & $F_{1}$ & $r=$ & $F:$ & LF & $\mathrm{Ao}_{1}$ & \\
\hline 1975 & 545.5 & 698.8 & 1509.8 & 1144.7 & 3898.8 & 1213.4 & 5113,2 \\
\hline $1978 \ldots$ & 300,0 & 678,2 & 596.2 & 814.2 & 2388.6 & 526,4 & 2915,0 \\
\hline 1979 & 322,4 & 486.6 & 706,6 & 906.1 & 2421.7 & 1000.8 & 3422,3 \\
\hline 1980 & 601,1 & & & & 2303.6 & 2874,0 & 5177,6 \\
\hline Moyenne & 442.2 & 621.2 & 937,5 & 955.0 & $\begin{array}{r}2753,2 \\
\pm 765 \\
\end{array}$ & $\begin{array}{r}1403,7 \\
+\quad 1021 \\
\end{array}$ & $\begin{array}{r}4156,9 \\
\pm 1160 \\
\end{array}$ \\
\hline tha $1 \ldots$ & & & & & 27,5 & 14,0 & 41,6 \\
\hline
\end{tabular}

Le stock de litière au sol varie entre 51,8 et 29,1 t ha ${ }^{-1}$ selon les années, avec une valeur moyenne de 41,6 tha 1 au cours de la période d'observation qui s'est étalée sur cinq années.

\subsection{La matière organique dans le sol}

Le premier mètre de sol renferme $105 \mathrm{t} \mathrm{ha}^{-1}$ de matière organique, dont plus de la moitié $\left(59,7 \mathrm{t} \mathrm{ha}^{-1}\right)$ est contenue dans les $20 \mathrm{~cm}$ correspondant à l'ancien fond de marais. La figure 2 indique la répartition verticale par tranches successives de $10 \mathrm{~cm}$ de ces $105 \mathrm{t}$ de matière organique.

Cette valeur peut être généralisće d'autant plus aisément à la station, que le substrat édaphique, constitué de sable, est très homogène. Même la présence d'un horizon organo-minéral, plus riche, n'influe pas sur cette homogénéité. Car si sa profondeur relative varie à cause du relief dunaire, son épaisseur, elle, est constante sur l'ensemble du site. 


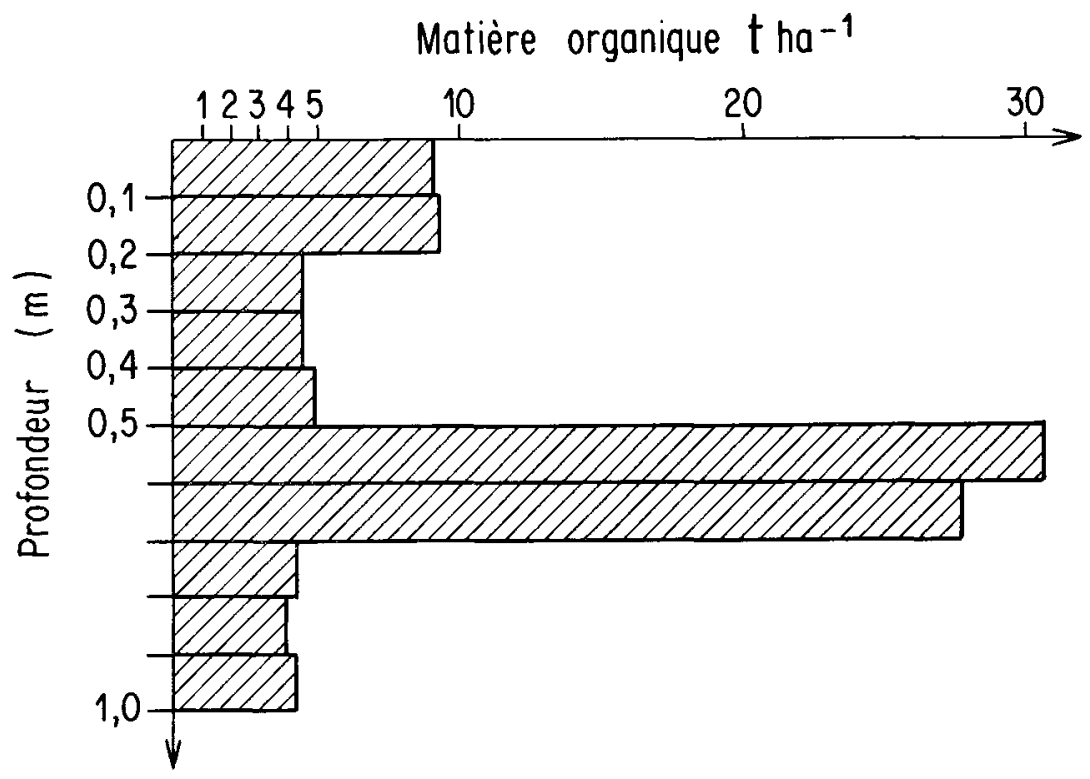

FIG. 2

Répartition de la matière organique par strates de sol sur un mètre de profondeur. Distribution of belowground organic matter.

\subsection{Les flux}

\subsection{L'accroissement annuel de phytomasse}

CABANettes \& Rapp (1981) ont établi les productions annuelles suivantes pour le peuplement étudié :

$\begin{array}{lr}\text { bois fort (tronc) } & 3,8 \mathrm{t} \mathrm{ha-1} \\ \text { menu bois (branches) } & 2,3 \\ \text { racines } & 1,1 \\ \text { aiguilles } & 7,4 \\ \text { fleurs mâles } & 0,3 \\ \text { cônes } & 3,7 \\ \quad \text { Total } & 18,6\end{array}$

La production globale de $18,6 \mathrm{t} \mathrm{ha}^{-1} \mathrm{an}^{-1}$ de ce peuplement est nettement supérieure à la production moyenne des forêts tempérées (PARde, 1980 ; LIETH \& MALAISSE, in LEMEE, 1974 ; ARts \& Marks, 1971 ; Satoo, 1970). Elle résulte essentiellement d'une très forte production foliaire, certainement en relation avec l'âge du peuplement. Par contre, à cause des conditions climatiques en région méditerranéenne, la production ligneuse est faible, par rapport aux autres essences forestières et à Pinus pinea L. sous d'autres latitudes (Cozzo, 1969). De même la production de graines est largement inférieure à celle constatée par DJAzIRI (1971) en Italie, qui avoisine $25 \mathrm{tha}^{-1} \mathrm{an}^{-1}$. 


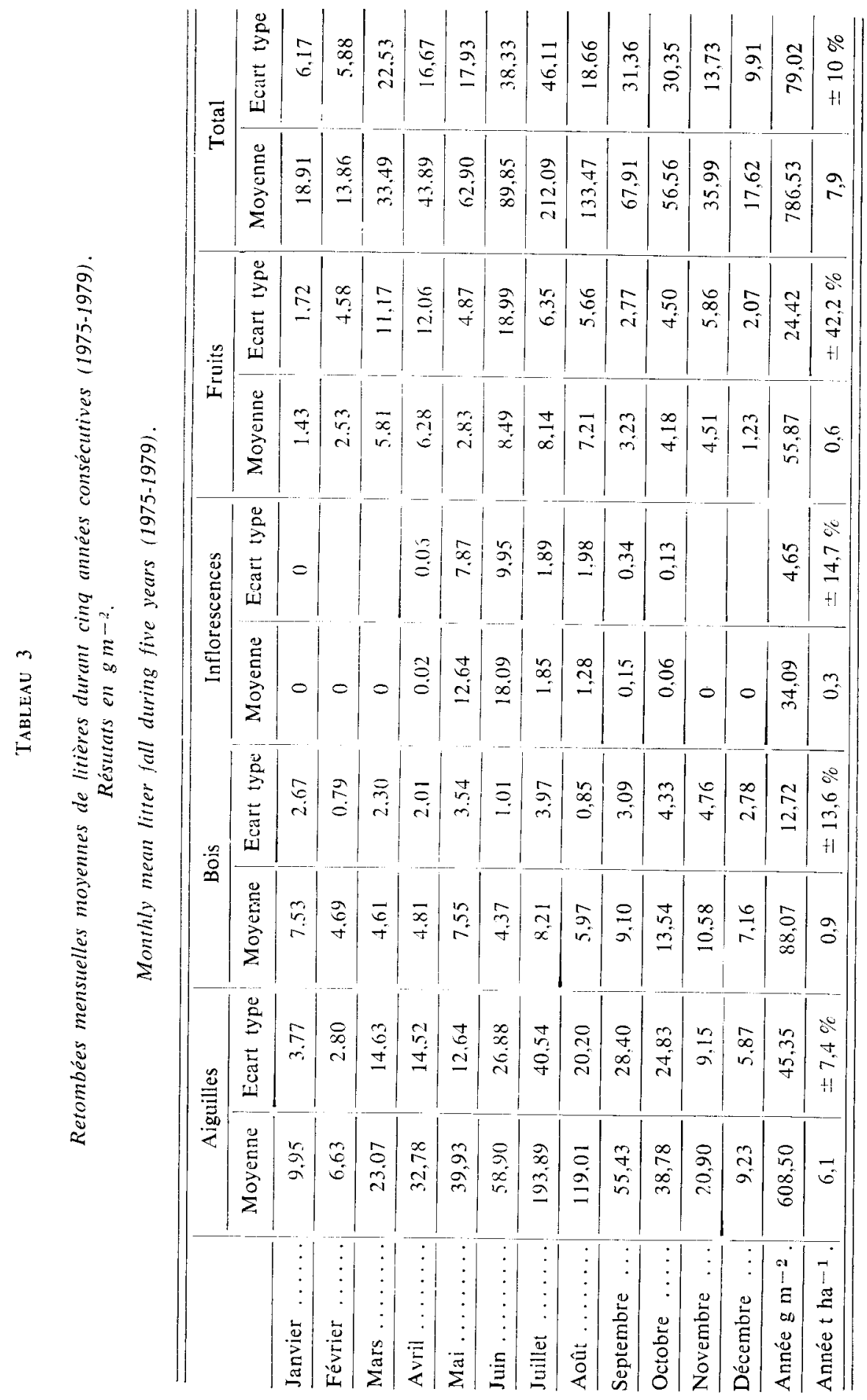




\subsection{La retombée de litière (tabl. 3)}

Au cours de cinq années consécutives d'observation, une moyenne de 7,9 t ha 1 de matière organique retourne annuellement au sol par l’intermédiaire de la litière de pin pignon. A cette quantité l'on peut ajouter 0.4 t ha ${ }^{1}$ an $^{-1}$ de litière provenant des autres espèces de la station, ce qui donne un apport global au sol de $8,3 \mathrm{t} \mathrm{ha-1}^{-1}$ an ${ }^{-1}$.

Les aiguilles, avec 6.1 tha 1 an 1 de valeur moyenne représentent à elles seules 77 p. 100 de la litière. Elles sont suivies du bois $\left(0,9 \mathrm{t} \mathrm{ha}^{-1}\right.$ an 1 ou 11,2 p. 100), des fruits $\left(0,6 \mathrm{t} \mathrm{ha}^{-1}\right.$ an 1 ou $\left.7,1 \mathrm{p} .100\right)$ et des inflorescences mâles $(0,3$ t ha-1 an 1 ou 4,3 p. 100). Les 0,4 tha 1 an $^{-1}$ d'apport supplémentaire représentent moins de 5 p. 100 de l'apport issu du pin pignon (tabl. 4).

\section{TABLFAU 4}

Part respective des différentes fractions de la litière en poids et en p. 100 durant les cinq amnées d'observation et valeur amnuelle moyente.

Respective proportions of the litter expressed as weight and as percent during five years of observation and mean values.

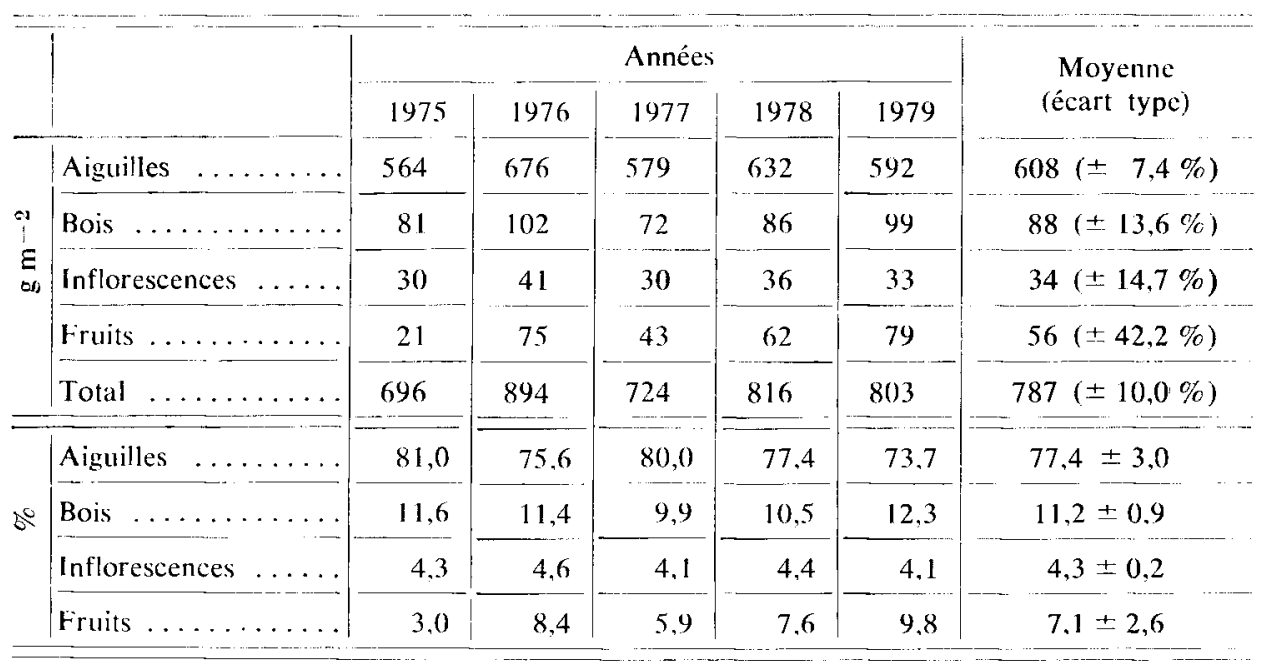

Durant les cinq années d'observation, les valeurs annuelles globales ont fluctué entre 7 et 8,91 ha $^{-1}$. Si ces fluctuations restent aléatoires au niveau de l'année, lon note une rythmicité mensuelle de la retombée qui se perpétue d'année en année (fig. 4). Le maximum mensuel de la chute de litière se situe toujours en juillet et 72 p. 100 de la litière totale, ou 76,8 p. 100 de la fraction aiguille, 99 p. 100 des inflorescences mâles et 53 p. 100 des cônes arrivent au sol entre mai et septembre.

Par contre la chute des brindilles ou de petites branches est alćatoire. Elle résulte en grande partic de l'interférence de perturbations physiques, tels des vents violents ou des orages et des pluies intenses. 


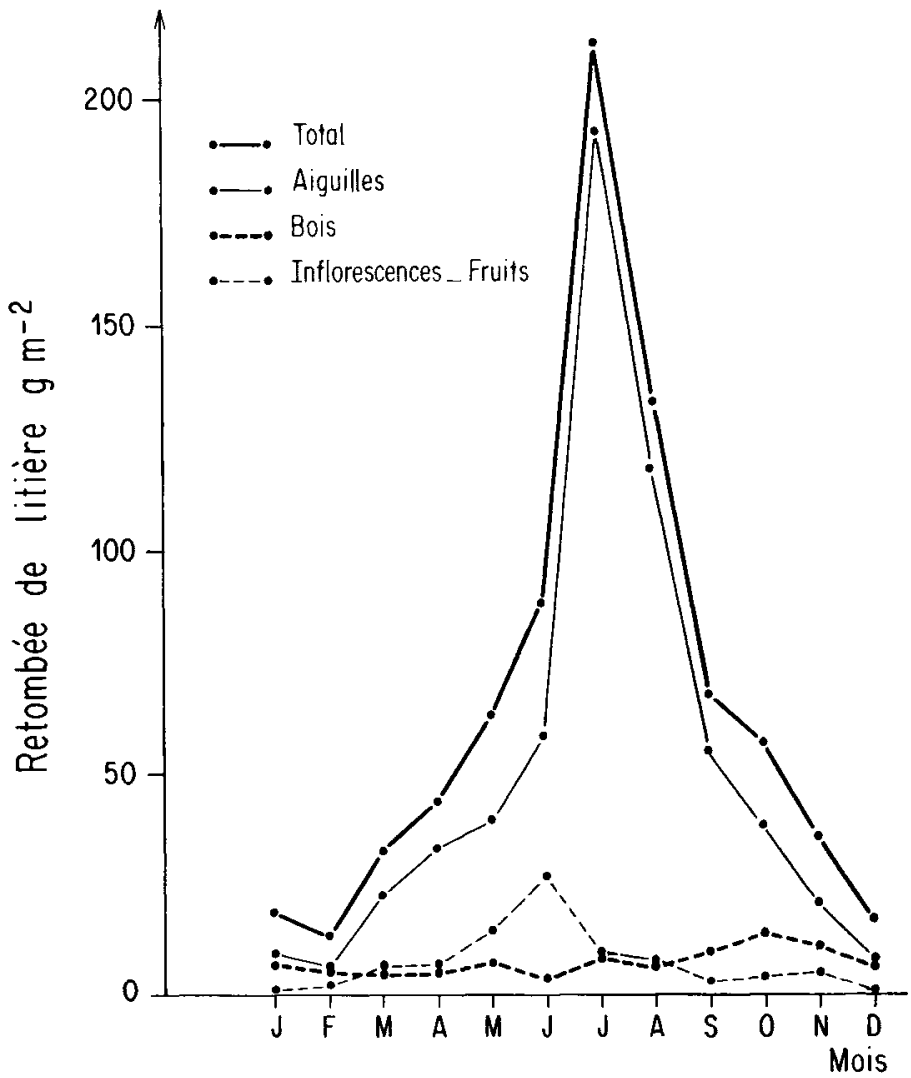

Fic; 3

Retombées moyennes mensuelles de la litière globale et des différentes fractions séparées. Résultats moyens de 5 années dobservation en $g m^{-?}$.

Average monthly litter fall during 5 years of observation.

La figure 3 représente la rythmicité mensuelle moyenne pour la période ḋobservation. Lion constate qu'elle est entièrement fonction de la retombée mensuelle des aiguilles. Lon peut noter à ce sujet une convergence dans la phénologie de deux pins méditerranéens assez courants dans le sud de la France : Pinus halepensis Mill. (RAPP, 1967) et Pinus pinea L. Dans les deux espèces, le maximum absolu de la chute des aiguilles se situe en juillet et plus des deux tiers retournent au sol entre mai et septembre. Cette chute se situe avant celle des aiguilles du pin sylvestre, qui se produit en août et septembre (BrAy \& Gorham, 1964). Cette sénescence précoce est probablement la consćquence directe de la sècheresse estivale en région méditerranéenne. Par la constitution d'un mulch, à la surface du sol, elle représente également une adaptation de la végétation à l'économie de l'eau de l'écosystème durant les mois les plus secs. 


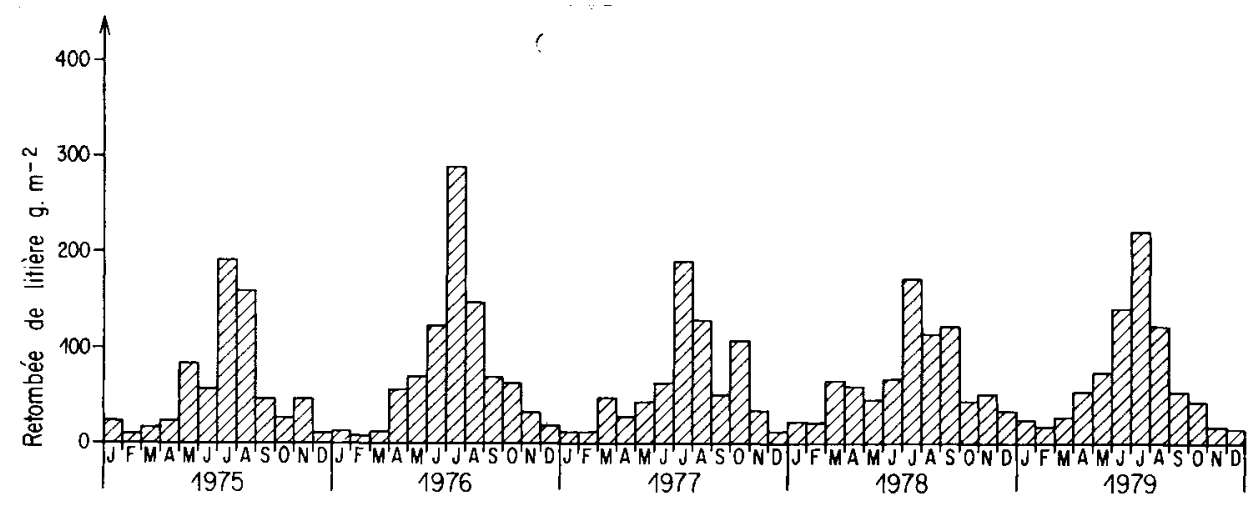

FIG. 4

Retombée mensuelle de litière au cours des cinq années d'observation.

Monthly litter fall during five years.

\subsection{La décomposition de la litière}

A partir des résultats des observations de la litière en place durant la période 1975-1980 et de ceux de la chute de litière au cours de la même période, l'estimation de la décomposition peut être entreprise par deux approches. L.une globale sur la période d'étude. l'autre pour certaines années spécifiques.

Si l'on compare le stock de litière au sol $\mathrm{L}$ aux temps $\mathrm{t}$ et $\mathrm{l}$ ' (exprimés par année) l'on peut écrire la relation :

$$
\mathrm{Lt}+\mathrm{A}-\mathrm{D}=\mathrm{Lt}^{\prime}
$$

A étant l'apport de litière fraîche durant $t^{\prime}$ - $t$ et $D$ représente la fraction de litière perdue durant le même laps de temps. Entre 1975 et 1980. le stock de litière au sol est passé de $51,1(\mathrm{Lt})$ à $51,8\left(\mathrm{Lt}^{\prime}\right) \mathrm{t} \mathrm{ha}^{-1}$. Au cours de cet intervalle de six ans, le sol a bénéficié d'un apport de $6 \times 7,9 \mathrm{t}^{-{ }^{-1}}$ de litière fraîche, soit $47,4 \mathrm{t}$ ha ${ }^{-1}$ (A).

L'on peut donc calculer :

$$
\begin{aligned}
\mathrm{D} & =51,1+47.4-51,8 \\
& =46,7 \mathrm{t} \text { ha } \cdots \text { en } 6 \text { ans d'où une perte moyenne annuelle de } 7.8 \mathrm{t} \text { ha }{ }^{t} .
\end{aligned}
$$

Par ailleurs Jenny et al. (1948) ont défini un coefficient de décomposition annuel de la litière à partir du stock de litière en place et de l'apport annuel de litière fraîche :

$$
K=\frac{100 A}{L+A}
$$

$A=$ apport de litière fraîche ;

$\mathrm{L}=$ stock de litière accumulée au sol. 
Pour les années 1977 et 1978 , nous avons respectivement :

$$
\begin{array}{ll}
1977 & K=\frac{720}{7,2+29,1}=19,8 \text { p. } 100 \\
1978 & K=\frac{810}{8,1+34,2}=19,1 \text { p. } 100
\end{array}
$$

ce qui donne un taux de décomposition moyen de 19.5 p. 100 . équivalent pour un stock de litière moyen de $41,6 \mathrm{t}$ ha-1 à $8,1 \mathrm{t}^{-1} \mathrm{a}^{-1} \mathrm{an}^{-1}$ de litière décomposée, quantité très proche de celle obtenue par la première approche $(7,8)$.

La première évaluation, obtenue sur toute la période d'étude peut être considérée comme la plus proche de la réalité. Elle se traduit par une légère augmentation du stock immobilisé, qui peut être considérée comme négligeable au niveau de ce genre d'étude.

Ces $7,8 \mathrm{t} \mathrm{ha}^{-1}$ de litière annuellement perdues sont à l'origine, pro-parte de la matière organique immobilisée dans le premier mètre du sol, sous forme de composés humiques, alors qu'une autre fraction retourne à l'atmosphère sous forme de $\mathrm{CO}$.

\section{Discussion}

La figure 5 synthétise et quantifie les différents paramètres de la distribution el des flux de matière organique à travers l'écosystème à Pinus pinea L. étudié.

Cet écosystène renferme 337 tha I de matière organique se répartissant en $179 \mathrm{t} \mathrm{ha}^{-1}(53,1$ p. 100) de phytomasse « vivante» et $158 \mathrm{t}$ ha 1 (46.9 p. 100) de matière «morte» recouvrant la nécromasse, la litière accumulée à la surface du sol et la matière organique propre atu sol.

La phytomasse produit chaque année l'équivalent de 10,4 p. 100 de sa masse (18.6 tha 1) de matéricl nouveau, dont $4 / 10$ de matériel pérenne $\left(7,2 \mathrm{t}^{-1}\right.$ ) et 6/10 (11,4 $\left.\mathrm{ha-1}^{-1}\right)$ d'organes photosynthétiques ou reproducteurs, à durée de vie limitće.

Globalement, les $7,9 \mathrm{t}^{\mathrm{h}} \mathrm{a}^{-1}$ de litière annuelle représentent 4,4 p. 100 de la phytomasse et les $7,2 \mathrm{t}$ ha ${ }^{1}$ d'accroissement pérenne $4 \mathrm{p}$. 100. Il y a done $3,5 \mathrm{tha} \mathrm{h}^{-1}$ de matière produite, équivalent à 2 p. 100 de la phytomasse qui ne sont pas comptabilisés dans le schéma. Ils correspondent en grande partie à deux points qui n'ont pas été étudiés avec précision dans ce travail. Le premier porte sur la mortalité et la décomposition des racines, qu`il est pratiquement impossible d'étudier en condition naturelle. On peut admettre qu'elle se situe légèrement en dessous du niveau de la production, done avoisiner 0,7 à 1 t ha 1 . La seconde incertitude porte sur la chute des cônes. En cffet, le dispositif de récolte de la retombée de litière n'est pas assez serré, pour permettre d'évaluer avec précision, la répartition spatiale très hétérogène de ces organes. D’ailleurs les résultats des observations et mesures, soit $0.6 \mathrm{t} \mathrm{ha}^{-1}$ de retombée annuelle moyenne de cônes, pour une production de 3,7 tha $\mathrm{a}^{-1}$ an ${ }^{-1}$ confirment amplement cette sous-estimation. 


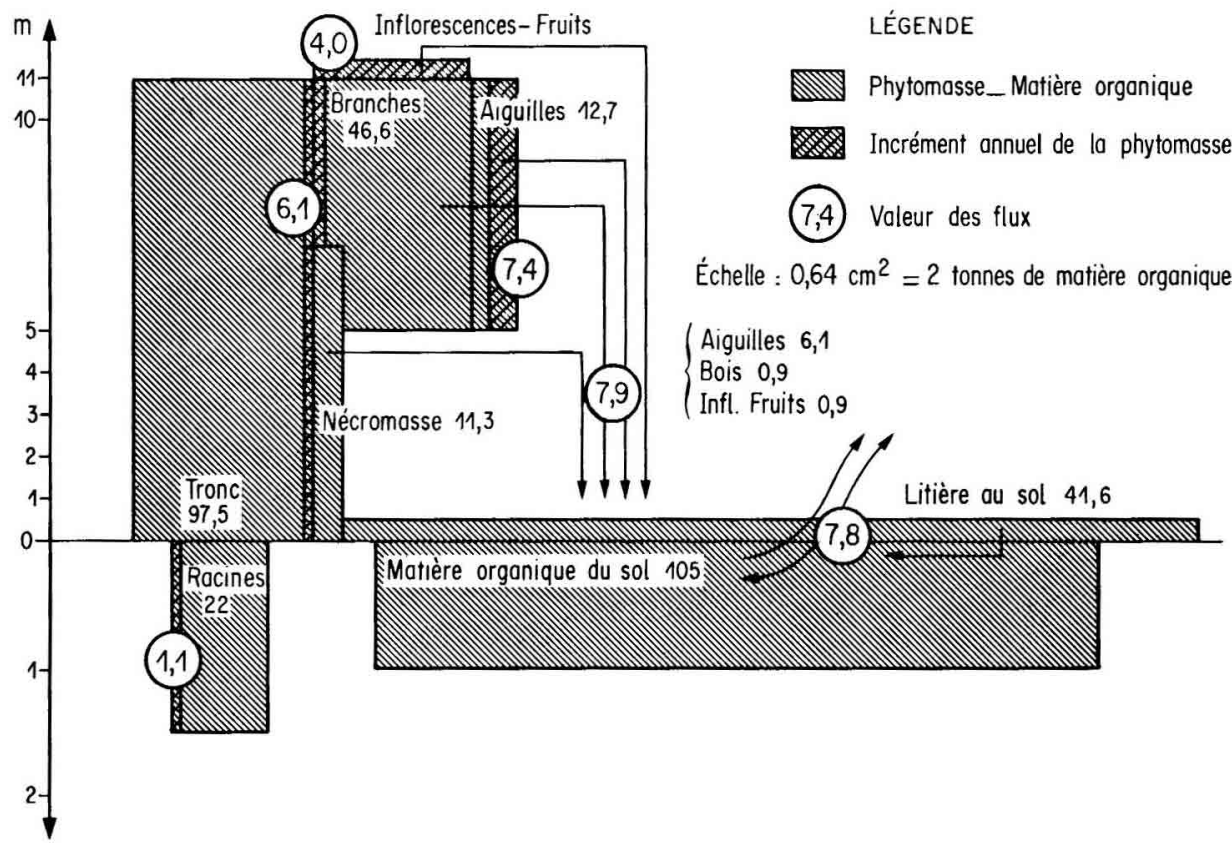

FIG. 5

Répartition et flux de la matière organique dans l'écosystème à pins pignons étudié. Résultats en $t$ ha-t.

Distribution and cycling of organic matter in a Pinus pinea L. stand. Standing crop values are in $t$ ha ${ }^{-1}$ and fluxes in $t$ ha- $t$ year-1.

Le matériel organique non inclus dans la phytocénose représente près de la moitié de la matière organique de l'écosystème. Les $2 / 3$ se trouvent dans la matière organique du sol, sur une tranche d'un mètre et ont subi de profondes 1ransformations par rapport au matériel originel pour former l'humus du sol. Seul le dernier tiers est représenté par du matériel végétal au sens strict (nécromasse) ou plus ou moins altéré (litière au sol).

Sur une longue période, la litière au sol fluctue très peu, alors qu'à court lerme, d'une année à l'autre, l'on peut observer des variations significatives de sa masse et donc de son taux de décomposition.

Ceci se confirme au niveau des entrées et des sorties de ce compartiment. L'apport moyen annuel de litière fraîche est de $7,9 \mathrm{t} \mathrm{ha}^{-1}$, alors que la décomposition moyenne est de $7,8 \mathrm{t} \mathrm{ha-1}^{-1}$ an- $^{-1}$. La masse immobilisée peut donc être considérée comme constante, ce qui indique que le système a atteint un stade d'équilibre non seulement au niveau de la litière où les entrées compensent les sorties, mais pour l'ensemble de la production, de la circulation et de la décomposition de la matière organique. 


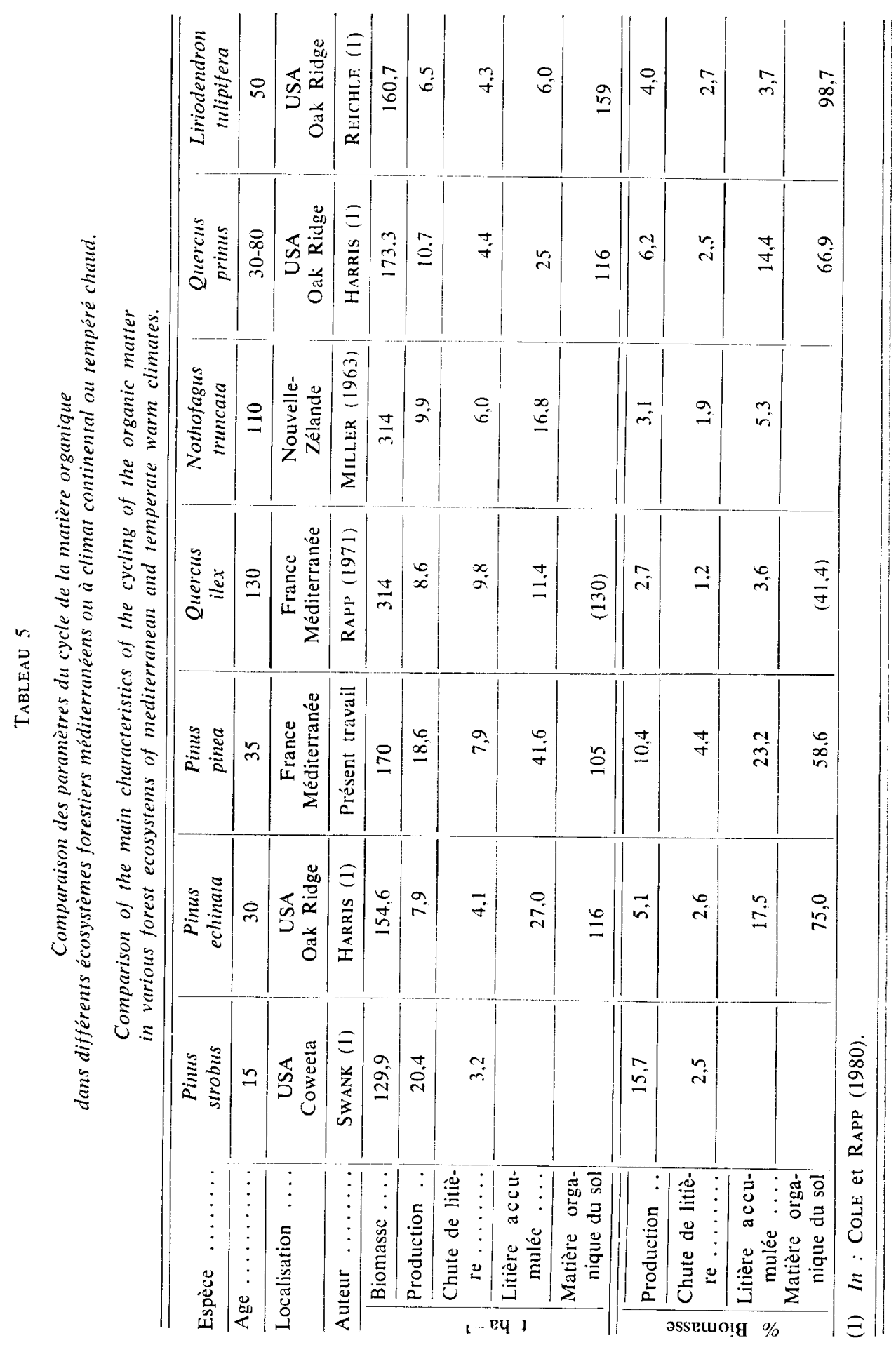


Tenant compte de cet état d'équilibre, l'on peut se poser la question du devenir des $7,8 \mathrm{t} \mathrm{ha}^{-1}$ an $^{-1}$ de matériel organique disparaissant de la litière. Schématiquement, une partie va être utilisée et réorganisée pour constituer le matériel humique du sol, une autre partie servira comme source énergétique pour ces transformations et pour la vie dans le sol et va être finalement restituée à l'atmosphère sous forme de dioxyde de carbone.

Mais les 105 t ha 1 de matière organique du substrat édaphique, sont elles aussi à peu près constantes, ceci d'autant plus que le système est stable. L'on peut donc admettre que la fraction de la litière, transformée en substances humiques, remplace du matériel humique plus ancien, qui est lui-même minéralisé. Ceci entraîne, au niveau du budget global de la matière organique de l'écosystème, une restitution vers l'atmosphère sous forme de gaz carbonique d'une quantité de $\mathrm{CO}_{2}$ équivalent sensiblement à $7,8 \mathrm{t} \mathrm{ha}^{-1}$ an $^{-1}$ de matière organique.

La comparaison de ces résultats avec ceux obtenus dans d'autres études est très difficile. En effet, ni les essences ou les caractéristiques sylviculturales, ni l'âge, ni les milicux édaphiques ou climatiques ne sont jamais semblables. le tableau 5 résume un certain nombre de paramètres pondéraux du cycle de la matière organique de groupements forestiers méditerranéens ou de climat continental ou tempéré chaud. Pour faciliter d'éventuelles comparaisons, l'on a également calculé la productivité primaire nette, la chute de litière, son immobilisation à la surface du sol et la réserve organique du sol par rapport à la phytomasse de l'écosystème. Ce tableau confirme bien la difficulté de telles comparaisons. Les rares relations susceptibles d'être constatées ou établies sont des généralités souvent banales et déjà largement connues.

Ainsi, la productivité plimaire représente selon l'essence, entre $15,7 \mathrm{p} .100$ et 2,7 p. 100 de la biomasse. Elle est surtout fonction de l'âge du peuplement, done d'un facteur essenticllement physiologique, de sa densité, donc des traitements sylvicoles, et probablement de l'alimentation en eau, facteur climatique et écologique. L'accumulation de la litière au sol est généralement plus élevée pour les résineux que pour les feuillus, ce qui cntraîne souvent une plus grande richesse en substances organiques du sol sous feuillus. L'on peut néanmoins remarquer que la station étudiée possède la plus faible réserve organique du sol, en raison essentiellement de la nature sableuse de son substrat édaphique, tout en présentant une bonne productivité. Ceci confirme le rôle éminemment favorable de l'alimentation hydrique de la phytocénose dans ce site (Iвrahim et al., 1982).

On peut encore ajouter que la masse de matière organique du sol qui provient de l'ancien marais ne représente pas une accumulation due au cycle de la matière organique de l'écosystème. Ceci entraîne deux hypothèses quant au cycle biogéochimique du système. Ou bien l'on a affaire à un blocage important, donc à une faible activité biologique et minéralisatrice au nivcau de la litièrc accumulée à la surface du sol, ou alors, l'activité biologique est très importante dans les horizons minéraux du sol. Une étude antérieure, portant sur le cycle de l'azote (RAPP et al., 1979) ferait plutôt pencher vers la première hypothèse. 


\section{Conclusions}

L'étude de la répartition et des flux de matière organique dans un peuplement de Pinus pinea L., âgé d'une trentaine d'années, indique les faits suivants :

- La biomasse s'élève à 179 tha '.

- Cette phytomasse produit chaque année $18.6 \mathrm{t}^{\text {ha-1 }}$ de matéricl nouveau : aiguilles, organes pérennes aériens et souterrains, organes reproducteurs.

- 7,9 t ha 1 de matériel retournent annuellement au sol par l'intermédiaire des litières. Lc maximum de retombée se situe entre juin et août.

- Cette litière est immobiliséc à la surface du sol pendant un certain laps de temps. Elle forme une couche organique de 7 à $12 \mathrm{~cm}$ d'épaisscur, correspondant à une masse moyenne de $41.6 \mathrm{tha}^{-}$'. qui fluctue d'une année à l'autre.

- Compte tenu de ce stock et de l'apport annuel de litière fraîche, on peut estimer les pertes de ce compartiment à 7.8 tha 1 an $\cdots 1$.

- Sur une profondeur d'un mètre, le sol renferme 105 t ha' 1 de substances organiques, dont la moitié ne provient pas du cycle biologique de l'écosystème mais d'un dépôt d'origine lagunaire.

- Pour l'ensemble de l'écosystème étudié, le stock de matière organique s’élève à $337 \mathrm{tha}$. dont 53.1 p. 100 sont contenus dans la biomasse alors que 46.9 p. 100 sont formés de matière organique morte ou recyclée par les diverses composantes du sous-système sol.

\section{Remerciements}

Lauteur tient à exprimer sa gratitude à M. A. Bi.ANCHARD pour sal collaboration technique. Celui-ci a notamment pris en charge bon nombre de relevés de terrain et le fastidieux travail de tri des échantillons. Ce travail a bénéficié du support matériel de la DGRST (décision d’ordre à la recherche 7872492).

\section{Summary}

Distribution and cycling of organic matter in a Pinus pinca L. stand

The distribution and turnover of organic matter has been studied in a thirty year old Pinus pinea L. stand, growing on the French mediterranean coast. The stand includes 800 stems per hectare with a mean height of 10.5 meters and a mean diameter at breast height $(\mathrm{DBH})$ of $20.2 \mathrm{~cm}$.

That stand included $337 \mathrm{t}$ ha 1 of organic matter. The above and below ground tree biomass amounted to $179 \mathrm{t}$ ha $\cdots 1$, the remaining $158 \mathrm{t}$ ha 1 being dead organic compounds. This last material could be subdivided as standing dead, litter and soil organic compounds.

The net primary production of the site amounted to $18.6 \mathrm{t}$ ha -1 of which $7.2 \mathrm{tha}-1$ are formed by perennial material. The yearly litter fall fraction amounted to 7.9 t ha 1 
(mean of a five year observation period). The litter layer on the soil surface amounted to $41.6 \mathrm{t} \mathrm{ha-1}$ and the losses accounted for $7.8 \mathrm{tha}^{-1}$ yearly. The soil organic matler on a one meter depth profile amounted to 105 t ha. 1 .

Regu le 15 novembre 1982.

Accepté le 2 mars 1983.

\section{Références bibliographiques}

ART H.W., Marks P.L., 1971. A summary table of biomass and net annual primary production in forest ecosystems of the world. In : Forest biomass stadies. Working Group on Forest Biomass Studies. Section 25, XVth I.U.F.R.O. Congress, Life Sciences and Agriculture Station, University of Maine at Orono, 3-32.

Bray J.R., Gorham E., 1964. Litter production in forests of the world. Adv. Ecol. Research, 2, 101-157.

Cabanetrtes A., 1979. Croissance, biomasse et productivité de Pinus pinca L. 'n Pefite Camargue. Thèse de 3" cycle, U.S.T.L. Montpellier, 175 p. (Ronéo.).

Cabanettes A., Rapp M., 1978. Biomasse, minéralomasse et productivité d'un écosystème à Pins pignons (Pinus pinea L.) du littoral méditerranéen. 1. Biomasse. Oicol. Plant., 13 (3), 271-286.

CabanetTes A., RaPP M., 1981. Biomasse, minćralomasse et productivité d'un écosystème à Pins pignons (Pinus pinea L.) du littoral méditerranéen. III. Croissance. Acta Ecol., aicol. Plant., 2 (16), (2), 121-136.

Cabanettes A., Rapl' M., 1981. Biomasse, minéralomasse et productivité d'un écosystème à Pins pignons (Pints pinea L.) du littoral méditerranéen. IV. Production. Acta Uicol. Ecol. Plant., 2 (16), (4), 381-394.

Cole D.W., Rapp M., 1980. Elemental cycling in forest ecosystems. In : Dynamic properties of forest ecosystems. D.E. Reichle (ed.), IPB 23, Cambridge Univ. Press, Cambridge, 341-409.

Cozzo D., 1969. Noticia sobre el crecimiento de Pints pinea en la provincia de Buenos Aires, Argentina. Rev. For. Argent., 13 (4), 119-124.

DJAZIRI A., 1971. Etude stationnelle du Pin pignon en Italie. Inst. Nat. Rech. Forest. de Tunisie, var. scientifique n" 9, 94 p.

Duvigneaud P., 1967. La productivite primaire des écosystèmes terrestres. In : Lanotite $M$. et Bourliêre F., Problèmes de Productivité Biologique. Masson et C', Paris, 246 p.

Emberger L., 1955. Une classification biogéographique des climats. Rev. Trav. Fac. Sci. Montpellier, Bot., 7, 3-43.

Iвraнiм M., 1979. Recherche sur la dynamique et le bilan de leau d'un écosystème ì Pin pignon (Pinus pinea L.) sur sable dunaire. Thèse d'Etat, U.S.T.L. Montpellier, 271 p. (Ronéo).

Ibrahim M., Rape M., 1979. Variations spatio-temporelles de la salinité du sol d'un pellplement de pins pignons (Pinus piner L.) du littoral méditeranéen. Ccol. Mediterranea., 4, 49-61.

Ibrahim M., Rapp M., Lossaint P., 1982. Economic de l'eau d'un écosystème à Pinus pinea L. du littoral méditerranéen. Ann. Sci. For., 39 (3), 289-306.

Jenny H., Gessel S.P., Bingham F.T., 1949. Comparative study of decomposition rates of organic matter in temperate and tropical region. Soil. Sci, 2, 419-432.

Lemee G., 1974. La productivité primaire de la forêt. $I n$ : Ecologie forestière, P. PrSson (ed.), Gauthier-Villars, Paris, 135-153.

Miller R.B., 1963. Plant nutrients in hand beech. N.Z.J. Sci., 6 (3), 365-413

Newbould P.J., 1967. Methods of estimating primary production of forests. IBP Handbook $n^{\circ} 2$, Blackwell, Scientific Publications, Oxford and Edinburgh, $62 \mathrm{p}$. 
Parde J., 1980. Forest biomass. For. Abstr., 41 (8), 343-362.

Rapp M., 1967. Production de litière et apport au sol d'éléments minéraux et d'azote dans un bois de Pins d'Alep (Pinus halepensis Mill.). Qcol. Plant., 2, 325-338.

RaPP M., 1971. Cycle de la matière organique et des éléments minéraux dans quelques écosystèmes méditerranéens. In : Ecologie du Sol. Recherche coopérative sur programme $n^{n}$ 40, C.N.R.S., Paris, vol. 2, 19-188.

Rapp M., Leclekc M.Cl., Lossaint P., 1979. - The nitrogen economy in a Pinus pinea L. stand. For. Ecol. Management, 2, 221-231.

Riedacker A., 1968. Méthodes indirectes d'estimation de la bionasse des arbres ei des peuplements forestiers. Doc. I.N.R.A.-C.N.R.F., Nancy, 24 p. (Ronéo).

Riedacker A., 1968. Méthodes d'estimation de la biomasse d'un arbre. Rapport stage D.E.A., Fac. Sci. Orsay, Doc. I.N.R.A.-C.N.R.F., Nancy, 40 p. (Ronéo.).

Rodin L.E., BAZILEVICH N.I., 1967. Production and mineral cycling in terrestrial vegetation. Oliver and Boyd, Edinburgh, 288 p.

Satoo T., SASSA T., 1970. Recherches sur la biomasse, l'accroissement et la perte par dessiccation des racines de Abies firma et Tsuga sieboldii. I.N.R.A., Station de Sylviculture et de Production, Doc., 79/07, 23 p. 